\title{
Heart Rate Variability: Impact of Differences in Outlier Identification and Management Strategies on Common Measures in Three Clinical Populations
}

\author{
KATHI J. KEMPER, CRAIG HAMILTON, MIKE ATKINSON
}

\begin{abstract}
Department of Pediatrics [K.J.K.], Department of Biomedical Engineering [C.H.], Wake Forest University Health Sciences, Winston-Salem, NC 27157; Research Department [M.A.], Institute of HeartMath, Boulder Creek, CA 95006
\end{abstract}

\begin{abstract}
Heart rate variability (HRV) is reported increasingly in pediatric research, but different strategies used to identify and manage potential outlier beats impact HRV parameter values in adults and animals. Do they in pediatrics? To compare the impact of different strategies to identifying and managing outliers, we used interbeat interval (IBI) data from three different populations: 10 stable premature infants, 33 stable pediatric oncology patients, and 15 healthy adults. Five commonly reported HRV parameters were compared using three identification and two management strategies to filter potential outliers. The three populations had different resting heart rates: $155 \pm 9$ beats per minute $(\mathrm{bpm})$ in infants, $105 \pm 17 \mathrm{bpm}$ in children, and $87 \pm 12 \mathrm{bpm}$ in adults. All three identification strategies flagged fewer than $2 \%$ of IBIs; the threshold identification strategy, excluding IBIs denoting heart rates $<30$ or $>300 \mathrm{bpm}$, identified significantly fewer outliers than the other two strategies and generated higher HRV parameters in all populations $(p<0.001)$. There were no significant differences in HRV parameters calculated by managing identified outliers by "tossing" them versus "interpolating" values. Different strategies for identifying potential outliers are associated with significant differences in HRV parameters. Pediatric researchers who report HRV should detail their outlier filtering strategies. (Pediatr Res 62: 337-342, 2007)
\end{abstract}

$\mathrm{H}^{2}$ eart rate variability (HRV) measurements are increasingly used by researchers to describe physiology, provide prognostic information, and measure response to therapies (1). HRV is the beat-to-beat variation of the interbeat interval (IBI) of the ECG (ECG). This variability is modulated primarily by the sympathetic and parasympathetic autonomic nervous system (2-4). Methods for calculating time and frequency-domain parameters of HRV were recommended by the Task Force of the European Society of Cardiology (5) and these methods have been widely adopted (6).

The SD of the IBI (SDNN) is the most widely used measure of HRV, followed by the root mean square of the difference of successive IBIs (RMSSD). Other commonly used parameters

Received March 12, 2007; accepted April 27, 2007.

Correspondence: Kathi J. Kemper, M.D., Caryl J. Guth, MD Chair for Holistic and Integrative Medicine, Professor, Pediatrics, Public Health Sciences and Family Medicine, Wake Forest University School of Medicine, Medical Center Boulevard, WinstonSalem, NC 27157; e-mail: kkemper@wfubmc.edu

The contents of this article are solely the responsibility of the authors and do not necessarily represent the official views of the NCCAM or the National Institutes of Health.

This project was made possible by grant numbers R21 AT001901 and by K24 AT002207 from NIH NCCAM. include pNN50 (the percentage of IBIs that differ by more than $50 \mathrm{~ms}$ from the previous IBI), and parameters from power spectral analyses based on fast Fourier transformations, particularly low frequency (LF) and high frequency (HF) power. HF oscillations $(0.15-0.4 \mathrm{~Hz})$ are thought to be markers of parasympathetic and particularly vagal autonomic tone. LF oscillations $(0.04-0.15 \mathrm{~Hz})$ reflect sympathetic and to a lesser extent, parasympathetic tone (7).

Increases in HRV parameters, up to a point, reflect adaptability, resilience and well being, while decreases in HRV reflect rigidity, poor health status and a poor prognosis in both adults and children with a wide variety of clinical conditions (8-23). In children HRV generally increases between the birth and fourteen years of age as the autonomic nervous system matures $(24,25)$. In adults HRV generally decreases with normal aging $(6,26,27)$. Therapeutic interventions such as exercise (28-30), meditation (31-33) and music (34) can increase HRV parameters. Given its correlation with clinical characteristics as well as its use as a prognostic tool and to measure response to therapies, studies reporting HRV have grown enormously since 1996.

As the use of HRV has grown, different researchers and software developers have used and recommended different strategies to identify and manage potential outliers observed in raw IBI data. Cardiologists and physiologists commonly recommend performing visual inspection of the ECG wave form, but some data collection devices used clinically report only IBI, making such inspection impossible. Also, visual inspection is costly in terms of time, particularly to review long recordings (more than several minutes in multiple patients). Some commercial software calculates HRV without telling users what method is used to deal with potential outliers; many publications fail to specify the outlier identification and management strategies used to filter the data before calculating HRV parameters.

Causes of outliers may vary in different populations. For example, in adults with heart disease, outliers may be due to

\footnotetext{
Abbreviations: bpm, beats per minute; HF, high frequency; HRV, heart rate variability; IBI, interbeat interval; Interp, interpolation; LF, low frequency; PctChg, percent change; pNN50, percentage of IBIs that differ by more than $50 \mathrm{msec}$ from the previous IBI; RMSSD, root mean square of the successive differences of the IBI of the ECG; SDChg, standard deviation change; SDNN, standard deviation of the beat to beat interval of the ECG
} 
premature ventricular contractions or other arrhythmias whereas in toddlers and preschool children in our experience movement artifacts predominate. Two methodological issues arise in dealing with IBIs that may be outliers: a) identification of those intervals and b) how to manage outliers once they have been identified. Strategies for identification of outliers have been published though they have not been used or reported consistently. These include intervals outside of a:

a) fixed range of acceptable interval values $(35,36)$; e.g., IBIs reflecting heart rates $<30$ or $>300 \mathrm{bpm}$; threshold rates vary depending on the clinical population of interest (which we refer to as "threshold" identification strategy);

b) selected percentage change from the mean of a set of previously accepted intervals $(3,13)$; e.g., if an IBI differs by more than $30 \%$ than the mean of the previous four intervals (which we refer to as "percent change" identification strategy);

c) change greater than a selected multiple of the SD of a set of previously accepted intervals $(37,38)$; e.g., more than five standard deviations from the mean of the previous 100 accepted intervals (which we refer to as "SD" identification strategy).

Once outliers are identified, there are also different strategies for managing them. The simplest approach is to "toss" that piece of data by removing it from the set $(13,38)$. On the other hand, some researchers replace the outlier with an interpolated value based on nearby accepted values (37,39-42).

Previous research in pigs $(43)$ and adults $(3,44)$ suggests that different outlier identification and management strategies might have important consequences when calculating HRV parameters. We conducted this study to compare the impact of different outlier management strategies on common HRV measures in three different human populations with different ages and resting heart rates: stable premature newborn infants, pediatric oncology patients, and healthy adults. We were particularly interested in whether the previous findings in animals and adults could be replicated in pediatric populations.

\section{METHODS}

Before conducting the main study, we wished to assess the correlation between IBI data collected by Minimitter with the gold standard of ECG data collected by Holter monitor. Therefore, we compared 15 min of simultaneous data recording using both devices. For this within study comparison, we included three healthy subjects ranging in age from 17 to $51 \mathrm{y}$ old who sat quietly during data collection. Pearson correlation coefficients were calculated for the IBI data from both systems for the raw (unfiltered data); data were filtered using the "percent change" strategy (using a difference of 30\% from the mean of the previous four intervals), interpolating suspected outliers; HRV parameters were then calculated separately at the Institute of HeartMath and at Wake Forest University School of Medicine.

For the main study, in the stable premature newborn infants, data were obtained from baseline measures collected for a study evaluating the impact of music on HRV. Infants were excluded if they had congenital heart disease, intracranial hemorrhage or were receiving antibiotics, pulmonary or cardiac medications. Subjects wore a MiniMitter $2000^{\mathrm{TM}}$ monitoring unit (MiniMitter Inc., Bend, OR), consisting of two ECG electrodes placed on the anterior chest wall, attached to a wireless transmitter unit. The Minimitter collects IBI data, sampling at $500 \mathrm{~Hz}$, and does not provide detailed ECG wave forms. All measurements were taken in the morning for at least $45 \mathrm{~min}$ starting at least
30 min after a baby had completed a feeding and 30 min before the next scheduled feeding.

For the pediatric oncology patients, patients were eligible if they were clinically stable and in the consolidation or maintenance phase of therapy for acute lymphocytic leukemia. The MiniMitter was also used, with data collected over 40 min during routine outpatient visits at the baseline visit for another study evaluating the impact of music on HRV. Data for both infants and children were collected by research assistants that were blind to the study hypotheses.

Data for healthy normal adults in normal sinus rhythm (sampled at a rate of $128 \mathrm{~Hz}$ on a Holter monitor) were obtained from the Physionet archives (45).

Data collection for premature infants, pediatric oncology patients and healthy older adults in the main study included at least 15 consecutive minutes of HRV monitoring. We picked the central $50 \%$ of the collected range to reduce artifacts from motion during monitor placement and removal.

Analysis software to implement and facilitate comparison of the various filtering strategies was developed using Matlab (The Mathworks, Natick, MA) based on standard calculations for SDNN, RMSSD, pNN50, LF, and LF. Three strategies for identification of IBI outliers were compared:

1. Threshold: if an IBI fell outside a range of $200-2000 \mathrm{~ms}$, corresponding to 30-300 bpm, which has been used in a previous study of low birthweight infants (36).

2. Percent change (PctChg): if an IBIs relative difference from the mean of the most recent four previously accepted intervals was greater than $30 \%$, which has been used in a previous study of pediatric oncology patients (13).

3. Standard deviation change (SDChg): if an IBI was greater than five standard deviations from the mean of the previously accepted 100 intervals, which was used in a previous study of premature infants (38).

Once outliers were identified by one of the three above criteria, two strategies for managing them were compared:

1. Removal from the data set (38), herein denoted as the "Toss" Method.

2. Replacement using cubic spline interpolation (39), herein denoted the "Interp" method, which replaces an outlier with a weighted average of nearby accepted IBI values.

Initialization of the PctChg identification strategy was determined by examining the first four values in a data set and comparing to a threshold range of one-half to twice the trimmed mean (mean of values excluding the upper and lower $20 \%$ of the values) of the data set, with a value outside this range being replaced with the trimmed mean. The SDChg identification strategy used a similar initialization method in which 100 values are initialized.

Power spectrum calculations were performed using Welch's method with a Hamming window of 1024 points, window overlap of 128, and an FFT of length the next power of 2 greater than the length of the data set. Data were initially resampled at $2 \mathrm{~Hz}$ to provide uniformly sampled data for the FFT calculation following which the Hamming window was applied to each window. Power was determined by integrating the range $0.04-0.15 \mathrm{~Hz}$ for LF and $0.15-0.4 \mathrm{~Hz}$ for $\mathrm{HF}$.

Group values were reported as mean \pm SE (SE). To compare the percent of outliers between the three identification strategies in each population, we used repeated measures analysis of variance. To compare differences between populations for each identification strategy, we applied analysis of variance and pair-wise comparisons. All HRV measures, except for SDNN, were log-transformed because of skewed distributions.

This study was approved by the Wake Forest University School of Medicine, WFUSM, Institutional Review Board.

\section{RESULTS}

Preliminary study. For the preliminary study comparing Holter with Minimitter data collection, the Pearson's r value for the raw data on IBI was 0.95 for one subject, 0.97 for another subject and 0.63 for the third subject. Raw data showed multiple potential outliers in the third subject's Minimitter data that were not apparent on the Holter data. After filtering using the SDChg identification and "Interp" strategies, HRV parameters calculated using the two data sources were virtually identical for all three subjects for mean HR, mean IBI and SDNN (Table 1). Calculations conducted by 
Table 1. Minimitter-versus Holter-generated HRV parameters

\begin{tabular}{|c|c|c|c|c|c|c|}
\hline \multirow[b]{2}{*}{ Parameter } & \multicolumn{2}{|c|}{$\begin{array}{c}\text { Subject } 1 \\
49 \text { year old female }\end{array}$} & \multicolumn{2}{|c|}{$\begin{array}{c}\text { Subject } 2 \\
17 \text { year old female }\end{array}$} & \multicolumn{2}{|c|}{$\begin{array}{c}\text { Subject } 3 \\
51 \text { year old male }\end{array}$} \\
\hline & MM & Holter & MM & Holter & MM & Holter \\
\hline Mean IBI (ms) & 833.6 & 833.6 & 1106 & 1106 & 920.1 & 920.3 \\
\hline SDNN (ms) & 26.9 & 28.6 & 123.4 & 123.9 & 33.0 & 34 \\
\hline Total Power & 222.1 & 231.8 & 3977 & 3951 & 328.5 & 330.2 \\
\hline
\end{tabular}

Table 2. Subject descriptions

\begin{tabular}{lccc}
\hline & Premature infants & Pediatric oncology & Healthy adults \\
& $\mathrm{N}=10$ & $\mathrm{~N}=33$ & 15 \\
\hline Age & $37+/-3$ weeks Range: 33 to 42 weeks & $10+/-5.0$ yrs Range: 9 months to 17 yrs & $66+/-4.2$ yrs Range: 62 to 76 yrs \\
Gender & $8(80 \%)$ male & $21(64 \%)$ male & $6(40 \%)$ male \\
Heart rate $(\mathrm{bpm})$ & $155 \pm 9$ & $105 \pm 17$ & $87 \pm 12$ \\
\hline
\end{tabular}

Heart rates were normally distributed; values reported as mean \pm standard deviation.

Institute of HeartMath (MA) and at WFUSM (CAH) on the filtered Minimitter data yielded identical HRV parameter values $(<1 \%$ difference for all parameters for all subjects; data not shown).

Subject description. For the main study, subjects included 10 newborns, 33 pediatric oncology patients and 15 healthy older adults (Table 2). The newborns' average heart rate during data collection was 155 beats per minute (bpm). The pediatric oncology patients had an average heart rate of 105 bpm. For the adults, the average heart rate was $87 \mathrm{bpm}$. Generally, the premature infants had the lowest values for HRV parameters (Table 2). The pediatric oncology patients had higher values than adults for all HRV parameters except SDNN.

Outlier identification. All three identification strategies flagged fewer than $2 \%$ of IBIs as outliers (Table 3 ). Although the percentage of outliers was quite low for all three strategies, there were significant differences between them given the large number of data points included in assessing heart rate over $15 \mathrm{~min}$. The Threshold strategy consistently identified significantly fewer IBIs as outliers (from $0.02 \%$ in infants to $0.12 \%$ in adults) than PctChg $(0.24 \%$ in infants to $1.98 \%$ in pediatric oncology patients) or SDChg identification strategies ( $0.81 \%$ in adults to $1.35 \%$ in infants) $(p<0.001)$.

Almost all the IBIs identified as outliers by Threshold were also identified by PctChg and SDChg methods. SDChg identified significantly more IBIs as outliers than the other strategies in infants and adults, but PctChg identified significantly more IBIs as outliers in children, several of whom had problems sitting still during data collection. Thus, the Threshold strategy seemed to identify a core group of outliers, while SDChg and PctChg identified slightly different groups of additional outliers in different populations.

The percentage of IBI identified as outliers was highest for pediatric oncology patients (who were observed to move most during data collection), but the percentage removed by any strategy even in this group was less than $2 \%$ (Table 2). The Threshold strategy identified fewer outliers than the other strategies. For example, among the infants, it flagged only $0.02 \%$ of the IBI, whereas, PctChy flagged $0.24 \%$ of IBI and
SDChg flagged $1.35 \%$ of IBI. Similarly, in pediatric oncology patients and adults, the Threshold strategy identified significantly fewer of the IBIs as outliers compared with other two identification strategies.

The Threshold strategy also generated the highest values for all five HRV parameters in all three clinical populations (Table 3). For example, among the premature infants, the RMSSD varied from 20.2 with Threshold to 7.6 with PctChg to 5.8 with SDChg, though all three strategies flagged less than $1.5 \%$ of IBI as outliers. In adults, the HF value dropped from 107.7 with the Threshold strategy to 29.8 with PctChg and 27.8 with SDChg, even though none of the three strategies identified more than $1 \%$ of IBIs as outliers. Thus, even quite small differences in the number of IBIs identified as outliers created substantial differences in HRV parameter values in all three patient populations.

Outlier management. Compared with the impact of different identification strategies, different management strategies had virtually no impact on HRV values (Table 3 ). For example, among premature infants using Threshold identification, the Toss and Interpolate methods yielded identical values for pNN50. Thus, after identifying outliers, the two management strategies yielded similar outcome values for all HRV parameters in all three patient populations.

\section{DISCUSSION}

This methodologic study evaluating the impact of IBI outlier identification and management strategies in two different pediatric and one adult population had three major findings. Data collected using Minimitter and Holter is comparable and HRV parameters calculated using our local software are nearly identical to those calculated at the Institute of HeartMath using different software. Differences in outlier identification strategies can significantly impact HRV values in pediatric patients as well as adults and animals. After controlling for the identification strategy, management by tossing versus interpolating data has minimal impact on HRV parameters.

All three strategies identified fewer than $2 \%$ of IBIs as outliers. The small differences in the number of outliers 
Table 3. HRV parameter valued for the three identification and two management strategies in three patient populations

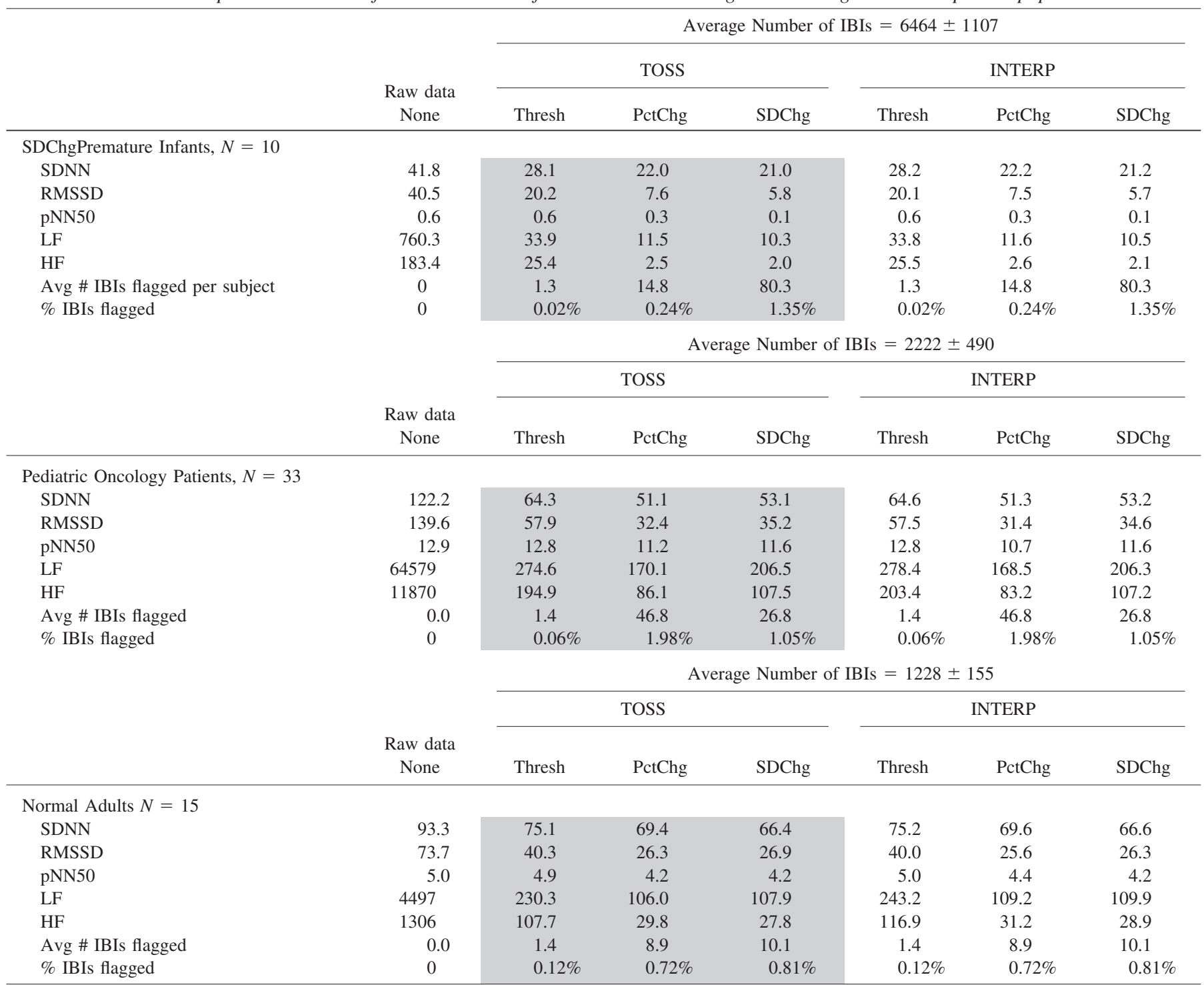

identified with the different strategies were associated with consistent, statistically significant differences in the values of commonly used HRV parameters. The Threshold strategy consistently identified fewer outliers, conserved the most data, and resulted in higher values for HRV parameters than the PctChg and SDChg methods in all three clinical populations. The results from different management strategies (tossing versus interpolating values) were minimal.

Our data on HRV parameters in populations with different ages and resting heart rates are consistent with other studies (1). For example, the values for HRV parameters were lower among premature infants than among the pediatric oncology patients, consistent with previous research showing increasing values of HRV parameters during childhood as the autonomic nervous system matures $(24,46)$. Similarly, the values for HRV parameters were generally higher among the 33 pediatric patients with an average age of $9.8 \mathrm{y}$ than in older adults with an average age of $66 \mathrm{y}$, consistent with previously observed declines in values of HRV parameters with aging $(1,47-49)$.
Furthermore, values for the HRV parameters in the pediatric patients in this study were similar to those of similar patients in other studies. For example, the HRV parameters among our premature infants were all somewhat lower than those reported by those evaluating HRV in term infants (50,51), consistent with reports that HRV parameters are lower among infants of lower postconceptional age $(21,22,25,36,50,52-54)$. Likewise, our results for the values of HRV parameters in pediatric oncology patients are similar to those found in earlier studies (13); values for HRV parameters in oncology patients may be lower than healthy children of similar ages due to cardiac toxicity of anthracycline chemotherapy, pain or other symptoms (55).

As the use of affordable devices for measuring IBI becomes more widespread, the issue of agreement between devices and analytic methods in determining HRV parameters becomes increasingly important. One study compared two devices (PowerLab ${ }^{\circledR}$ with Chart ${ }^{\circledR}$ software and PolarTransmitter Advantage ${ }^{\circledR}$ with PrecisionPerformance ${ }^{\circledR}$ software) among 36 healthy adults (mean age $27 \mathrm{y}$ ) during breathing with a 
metronome; this method resulted in excellent agreement between the two devices (56), but this level of agreement may not be found in patients with arrhythmias or movement artifacts in the HRV recordings.

Our results confirm and extend the findings of earlier studies evaluating a) the impact of outlier management on HRV parameters in healthy pigs (43) and b) a the impact of different strategies for editing IBI data in adults with and without heart disease (44). The animal study compared HRV parameters generated from a gold-standard ECG recording with those generated from a device measuring IBI. Fewer than $0.1 \%$ of total IBI were identified as anomalies or outliers, but this small number of outliers had dramatic impact on the values of HRV parameters, increasing SDNN and RMSSD values by $45 \%$ to $50 \%$; anomalies spuriously increased the values of total and parasympathetic activity as well (43). The authors concluded that even a small proportion of outliers can bias the outcome of HRV parameters, but that correcting the errors through careful identification could allow the use of IBI collection devices interchangeably with ECG. Similarly, in the study of adults with and without heart disease, different editing strategies resulted in "remarkably" different effects on HRV parameters (44). These conclusions support our finding that small differences in IBI identification can significantly impact HRV parameters in three different human clinical populations.

Our study had several limitations. Minimitter data collection did not allow visual inspection of the ECG data for "gold standard" outlier identification; outliers were not classified as to their cause (movement artifact versus ectopy, arrhythmia or device error). Although visual inspection is desirable, our data collection methods in the pediatric groups are likely to be similar to other clinical researchers who use relatively inexpensive IBI recording devices. Despite the small sample size and use of different data collection instruments in the adults and pediatric groups, which might have been expected to bias the results toward the null hypothesis, comparison of the different outlier identification strategies demonstrated significant differences that were robust across ages and health status in pediatric patients as well as adults. The impact of these different strategies on 24 -h recordings remains to be determined in future studies.

As Malik et al. noted, "filtering and editing of the computer-recognized RR interval sequence" are necessary to remove artifacts from beat recognition errors, movement and recording artifacts and arrhythmias (3). Researchers who compare HRV data over time in response to different therapeutic interventions or in different populations need to be aware of the impact different outlier handling strategies can have on the values of commonly used HRV parameters. Clinicians and researchers who report HRV parameters should clearly specify their outlier identification and management strategies to facilitate meaningful comparisons.

Acknowledgments. We are grateful to Debbie Dirkse and Teresa Shaw for collecting HRV data in premature infants and pediatric oncology patients. We appreciate advice from Dr. Ary Goldberger and data on healthy adults from the Arrhythmia
Monitoring Laboratory of the Margaret and H.A. Rey Institute for Nonlinear Dynamics in Medicine. We are also grateful to Paula Stant for her assistance in manuscript preparation.

\section{REFERENCES}

1. Acharya UR, Kannathal N, Sing OW, Ping LY, Chua T 2004 Heart rate analysis in normal subjects of various age groups. Biomed Eng Online 3:24

2. 1996 Heart rate variability. Standards of measurement, physiological interpretation, and clinical use. Task Force of the European Society of Cardiology and the North American Society of Pacing and Electrophysiology. Eur Heart J 17:354-381

3. Malik M, Farrell T, Cripps T, Camm AJ 1989 Heart rate variability in relation to prognosis after myocardial infarction: selection of optimal processing techniques. Eur Heart J 10:1060-1074

4. McCraty R, Atkinson M, Tiller WA, Rein G, Watkins AD 1995 The effects of emotions on short-term power spectrum analysis of heart rate variability. Am J Cardiol 76:1089-1093

5. 1996 Heart rate variability: standards of measurement, physiological interpretation and clinical use. Task Force of the European Society of Cardiology and the North American Society of Pacing and Electrophysiology. Circulation 93:1043-1065

6. Kleiger RE, Stein PK, Bigger JTJr 2005 Heart rate variability: measurement and clinical utility. Ann Noninvasive Electrocardiol 10:88-101

7. Azad N, Byszewski A, Sarazin FF, McLean W, Koziarz P 2003 Hospitalized patients' preference in the treatment of insomnia: pharmacological versus nonpharmacological. Can J Clin Pharmacol 10:89-92

8. Goldberger AL, Peng CK, Lipsitz LA 2002 What is physiologic complexity and how does it change with aging and disease? Neurobiol Aging 23:23-26

9. Bigger JTJr., Fleiss JL, Rolnitzky LM, Steinman RC 1992 Stability over time of heart period variability in patients with previous myocardial infarction and ventricular arrhythmias. The CAPS and ESVEM investigators. Am J Cardiol 69:718-723

10. Hirvonen HE, Salmi TT, Heinonen E, Antila KJ, Valimaki IA 1989 Vincristine treatment of acute lymphoblastic leukemia induces transient autonomic cardioneuropathy. Cancer 64:801-805

11. Morrow GR, Andrews PL, Hickok JT, Stern R 2000 Vagal changes following cancer chemotherapy: implications for the development of nausea. Psychophysiology 37:378-384

12. Takeshita A, Uehara A, Shinjo K, Naito K, Sahara N, Yamazaki K, Katoh H, Kamikawa T, Ohnishi K, Maekawa M, Hayashi H, Ohno R 2004 Impairment of heart rate variability control during arsenic trioxide treatment for acute promyelocytic leukemia. Leukemia 18:647-648

13. Kemper KJ, Woods CR, Yard B, Cohen DG, McLean T, Atkinson M 2004 Heart rate variability in pediatric patients with leukemia: A brief report. J Cancer Int Med 2:137-143

14. Stein PK, Kleiger RE 1999 Insights from the study of heart rate variability. Annu Rev Med 50:249-261

15. Katon WJ 2003 Clinical and health services relationships between major depression, depressive symptoms, and general medical illness. Biol Psychiatry 54:216-226

16. van Dijk M, de Boer JB, Koot HM, Duivenvoorden HJ, Passchier J, Bouwmeester N, Tibboel D 2001 The association between physiological and behavioral pain measures in 0- to 3-year-old infants after major surgery. J Pain Symptom Manage 22:600-609

17. Biswas AK, Scott WA, Sommerauer JF, Luckett PM 2000 Heart rate variability after acute traumatic brain injury in children. Crit Care Med 28:3907-3912

18. Goldstein B, Fiser DH, Kelly MM, Mickelsen D, Ruttimann U, Pollack MM 1998 Decomplexification in critical illness and injury: relationship between heart rate variability, severity of illness, and outcome. Crit Care Med 26:352-357

19. Grunau RE, Oberlander TF, Whitfield MF, Fitzgerald C, Lee SK 2001 Demographic and therapeutic determinants of pain reactivity in very low birth weight neonates at 32 Weeks' postconceptional Age. Pediatrics 107:105-112

20. Hanna BD, Nelson MN, White-Traut RC, Silvestri JM, Vasan U, Rey PM, Patel MK, Comiskey E 2000 Heart rate variability in preterm brain-injured and very-low-birthweight infants. Biol Neonate 77:147-155

21. Doussard-Roosevelt JA, Porges SW, Scanlon JW, Alemi B, Scanlon KB 1997 Vagal regulation of heart rate in the prediction of developmental outcome for very low birth weight preterm infants. Child Dev 68:173-186

22. Cabal LA, Siassi B, Zanini B, Hodgman JE, Hon EE 1980 Factors affecting heart rate variability in preterm infants. Pediatrics 65:50-56

23. Griffin MP, O'Shea TM, Bissonette EA, Harrell FE Jr, Lake DE, Moorman JR 2004 Abnormal heart rate characteristics are associated with neonatal mortality. Pediatr Res 55:782-788

24. Kazuma N, Otsuka K, Wakamatsu K, Shirase E, Matsuoka I 2002 Heart rate variability in normotensive healthy children with aging. Clin Exp Hypertens $24: 83-89$

25. Massin M, von Bernuth G 1997 Normal ranges of heart rate variability during infancy and childhood. Pediatr Cardiol 18:297-302

26. Agelink MW, Malessa R, Baumann B, Majewski T, Akila F, Zeit T, Ziegler D 2001 Standardized tests of heart rate variability: normal ranges obtained from 309 healthy humans, and effects of age, gender, and heart rate. Clin Auton Res 11:99-108

27. Yeragani VK, Sobolewski E, Kay J, Jampala VC, Igel G 1997 Effect of age on long-term heart rate variability. Cardiovasc Res 35:35-42

28. Stein PK, Ehsani AA, Domitrovich PP, Kleiger RE, Rottman JN 1999 Effect of exercise training on heart rate variability in healthy older adults. Am Heart J 138:567-576

29. Sandercock GR, Bromley PD, Brodie DA 2005 Effects of exercise on heart rate variability: inferences from meta-analysis. Med Sci Sports Exerc 37:433-439 
30. Tsai MW, Chie WC, Kuo TB, Chen MF, Liu JP, Chen TT, Wu YT 2006 Effects of exercise training on heart rate variability after coronary angioplasty. Phys Ther 86:626-635

31. Kubota Y, Sato W, Toichi M, Murai T, Okada T, Hayashi A, Sengoku A 2001 Frontal midline theta rhythm is correlated with cardiac autonomic activities during the performance of an attention demanding meditation procedure. Brain Res Cogn Brain Res 11:281-287

32. Peng CK, Mietus JE, Liu Y, Khalsa G, Douglas PS, Benson H, Goldberger AL 1999 Exaggerated heart rate oscillations during two meditation techniques. Int J Cardiol 70:101-107

33. Paul-Labrador M, Polk D, Dwyer JH, Velasquez I, Nidich S, Rainforth M, Schneider R, Merz CN 2006 Effects of a randomized controlled trial of transcendental meditation on components of the metabolic syndrome in subjects with coronary heart disease. Arch Intern Med 166:1218-1224

34. Iwanaga M, Kobayashi A, Kawasaki C 2005 Heart rate variability with repetitive exposure to music. Biol Psychol 70:61-66

35. Yum MK, Kim JH 2003 A very-short-term intermittency of fetal heart rates and developmental milestone. Pediatr Res 53:915-919

36. Sahni R, Schulze KF, Kashyap S, Ohira-Kist K, Fifer WP, Myers MM 2000 Maturational changes in heart rate and heart rate variability in low birth weight infants. Dev Psychobiol 37:73-81

37. Persson H, Kumlien E, Ericson M, Tomson T 2005 Preoperative heart rate variability in relation to surgery outcome in refractory epilepsy. Neurology 65:1021-1025

38. Griffin MP, Moorman JR 2001 Toward the early diagnosis of neonatal sepsis and sepsis-like illness using novel heart rate analysis. Pediatrics 107:97-104

39. Barnaby D, Ferrick K, Kaplan DT, Shah S, Bijur P, Gallagher EJ 2002 Heart rate variability in emergency department patients with sepsis. Acad Emerg Med 9:661-670

40. McNames J, Thong T, Aboy M 2004 Impulse rejection filter for artifact removal in spectral analysis of biomedical signals. Conf Proc IEEE End Med Biol Soc 1:145-148

41. Bennett AJ, Sponberg AC, Graham T, Suomi SJ, Higley JD, DePetrillo PB 2001 Initial ethanol exposure results in decreased heart rate variability in ethanol-naive rhesus monkeys. Eur J Pharmacol 433:169-172

42. Thong T, McNames J, Aboy M, Goldstein B 2004 Prediction of paroxysmal atrial fibrillation by analysis of atrial premature complexes. IEEE Trans Biomed Eng 51:561-569

43. Marchant-Forde RM, Marlin DJ, Marchant-Forde JN 2004 Validation of a cardiac monitor for measuring heart rate variability in adult female pigs: accuracy, artefacts and editing. Physiol Behav 80:449-458
44. Salo MA, Huikuri HV, Seppanen T 2001 Ectopic beats in heart rate variability analysis: effects of editing on time and frequency domain measures. Ann Noninvasive Electrocardiol 6:5-17

45. Goldberger AL, Amaral LA, Glass L, Hausdorff JM, Ivanov PC, Mark RG, Mietus JE, Moody GB, Peng CK, Stanley HE 2000 PhysioBank, PhysioToolkit, and PhysioNet: components of a new research resource for complex physiologic signals. Circulation 101:E215-E220

46. Silvetti MS, Drago F, Ragonese P 2001 Heart rate variability in healthy children and adolescents is partially related to age and gender. Int J Cardiol 81:169-174

47. Choi JB, Hong S, Nelesen R, Bardwell WA, Natarajan L, Schubert C, Dimsdale JE 2006 Age and ethnicity differences in short-term heart-rate variability. Psychosom Med 68:421-426

48. Corino VD, Matteucci M, Cravello L, Ferrari E, Ferrari AA, Mainardi LT 2006 Long-term heart rate variability as a predictor of patient age. Comput Methods Programs Biomed 82:248-257

49. Beckers F, Verheyden B, Aubert AE 2006 Aging and nonlinear heart rate control in a healthy population. Am J Physiol Heart Circ Physiol 290:H2560-H2570

50. Longin E, Schaible T, Lenz T, Konig S 2005 Short term heart rate variability in healthy neonates: normative data and physiological observations. Early Hum Dev 81:663-671

51. Mehta SK, Super DM, Connuck D, Salvator A, Singer L, Fradley LG, Harcar-Sevcik RA, Kirchner HL, Kaufman ES 2002 Heart rate variability in healthy newborn infants. Am J Cardiol 89:50-53

52. Feldman R, Eidelman AI 2003 Skin-to-skin contact (Kangaroo Care) accelerates autonomic and neurobehavioural maturation in preterm infants. Dev Med Child Neurol 45:274-281

53. Galland BC, Taylor BJ, Bolton DP, Sayers RM 2006 Heart rate variability and cardiac reflexes in small for gestational age infants. J Appl Physiol 100:933-939

54. Rosenstock EG, Cassuto Y, Zmora E 1999 Heart rate variability in the neonate and infant: analytical methods, physiological and clinical observations. Acta Paediatr 88:477-482

55. Postma A, Elzenga NJ, Haaksma J, Schasfoort-Van Leeuwen MJ, Kamps WA, Bink-Boelkens MT 2002 Cardiac status in bone tumor survivors up to nearly 19 years after treatment with doxorubicin: a longitudinal study. Med Pediatr Oncol 39:86-92

56. Radespiel-Troger M, Rauh R, Mahlke C, Gottschalk T, Muck-Weymann M 2003 Agreement of two different methods for measurement of heart rate variability. Clin Auton Res 13:99-102 